\title{
9 Rahmenkonzept (conceptual framework)
}

\subsection{Begründung und Aufgabenstellung}

Warum ein Rahmenkonzept? Vordergründig könnte der Eindruck entstehen, dadurch würde alles nur noch komplizierter, als es sowieso schon ist - warum den langen Weg gehen, wenn der Kürzere doch vorgezeichnet erscheint: Qualitätssicherung und Qualitätsmanagement sind in Deutschland etabliert, die Thematik Patientensicherheit ist integriert, Instrumente zur Verbesserung der Transparenz einschließlich Public Reporting wurden eingeführt, da führt doch kein Weg daran vorbei, als nächstes Pay for Performance, die qualitätsbezogene Vergütung einzuführen. Wie in Kapitel 8.1 bereits angemerkt: Hauptsache, es geht weiter.

Vielleicht ist dies auch richtig, vielleicht ist $\mathrm{P}_{4} \mathrm{P}$ wirklich der nächste Schritt. Allerdings ist dieses Vorgehen riskant, denn bei Kopplung von Vergütungsbestandteilen an Qualitätsindikatoren wird die Sache kritisch - ein Misslingen wäre fatal. Dazu kommen andere Faktoren, z.B. dass sich im deutschen Gesundheitswesen doch eine erhebliche Anzahl von Detailregelungen „angesammelt" hat, die alle das Thema Qualität betreffen, jedoch häufig den Eindruck einer dem Druck des gesetzgeberischen Moments geschuldeten Kleinteiligkeit machen (zur Übersicht s. Kap. 7). Auch kann nicht von der Hand gewiesen werden, dass bei der Vielfältigkeit der Problemstellungen im Bereich Qualität und Patientensicherheit eine übergeordnete „umfassende Qualitätsstrategie“ in Deutschland bislang fehlt. Allerdings ist eine solche 
umfassende Qualitätsstrategie nur zu entwickeln, wenn ein problemorientiertes Qualitätsverständnis zugrunde gelegt wird, das dem deutschen Gesundheitswesen angemessen ist und die Entwicklungsnotwendigkeiten wiedergibt.

Wenn man einen großen Schritt zurücktritt, kann man es auch so sagen: eine umfassende Qualitätsstrategie und jedes der eingesetzten Instrumente wie $\mathrm{P} 4 \mathrm{P}$ oder Public Reporting sind selbst komplexe Interventionen, und zwar ihrerseits Interventionen in einem überaus komplexen System. Komplexe Interventionen in einem komplexen System, eine solche Situation ist ohne ein theoretisches Grundkonzept nicht zu meistern (vgl. Campbell et al. 20oo, MRC 20oo, s. Abb. 45). Anderenfalls wäre die Einführung nicht adäquat zu gestalten, und die Erwartungen an die Wirksamkeit wären nicht in einem realistischen Rahmen zu halten (zum Begriff der doppelten Komplexität s. Kap. 8.3.2). Die P4P-Programme in den USA und Großbritannien sind ein gutes Beispiel: man hat viel erwartet („Qualität statt Menge finanzieren“), die frühen Ergebnisse waren noch recht positiv (Lindenauer et al. 2007), aber dann folgte die Ernüchterung (zur Darstellung der langfristigen Effekte s. Kap. 6.4).

Warum? Der wichtigste Grund dürfte darin liegen, dass man Indikatoren verwendet hat, die z.T. schon Jahrzehnte im Gebrauch waren, schon in den Public Reporting Programmen verwendet worden waren (dual use), die also schon allseits bekannt und „eingeübt" waren, und die dann (vorhersehbarer Weise, würde man heute sagen) keinen weiteren Effekt mehr zeigen konnten. Und genau der gleichen Gefahr sehen wir uns heute in Deutschland gegenüber, nämlich die altbekannten Indikatoren aus der Qualitätssicherung nach $\$ 137$ einfach finanziell aufzuladen, als P4P-Programm zu bezeichnen - und die Enttäuschung wird riesengroß sein.

Ein Rahmenkonzept, das den Hintergrund dafür bildet, dass solche Fehlentwicklungen vermieden werden, muss in der Lage sein,

\begin{tabular}{|c|c|}
\hline Theory/hypothesis & $\begin{array}{l}\text { - Theoretical basis for an intervention e.g., theories of organisational change } \\
\text { - Evidence by previous studies }\end{array}$ \\
\hline Phase 1: Modelling & $\begin{array}{l}\text { - Identifying components of intervention and underlying mechanisms } \\
\text { e.g., qualitative research, case studies }\end{array}$ \\
\hline Phase 2: Exploratory trial & $\begin{array}{l}\text { - Defining intervention and trial design } \\
\text { - Piloting outcome measures e.g., qualitative research, case studies }\end{array}$ \\
\hline Phase 3: RX trial & $\begin{array}{l}\text { - Adequately controlled, randomised trial } \\
\text { - Reproducible, appropriate power }\end{array}$ \\
\hline Phase 4: long term & $\begin{array}{l}\text { - Effective implementation of intervention } \\
\text { - Observation, adverse events }\end{array}$ \\
\hline
\end{tabular}

Abb. 45 Die Evaluation einer (umfassenden) Qualitätsstrategie sowie seiner Instrumente ist eine komplexe Intervention, die einer theoretischen Basis bedarf. 
- die Vielfalt der Faktoren, die auf das Verhalten der Leistungsanbieter einwirken und den Effekt von qualitätsverbessernden Instrumenten (z.B. P4P) mit beeinflussen, zu berücksichtigen,

- das Gesundheitssystem als Ganzes in seiner Reaktion auf qualitätsverbessernde Instrumente (z.B. P4P) mit einzubeziehen,

- die Situationen, in denen qualitätsverbessernde Instrumente (z.B. P4P) als wirksames Instrument sinnvoll einzusetzen sind, zu spezifizieren (Priorisierung),

- die methodischen Anforderungen an die Gestaltung des Instrumentes zu charakterisieren,

- die eventuell auftretenden negativen Konsequenzen zu antizipieren, damit hier frühzeitig Gegenmaßnahmen getroffen werden können,

- besonders bei $\mathrm{P}_{4} \mathrm{P}$ das Zusammenspiel mit anderen Vergütungssystemen und anderen qualitätsrelevanten Entwicklungen im Gesundheitswesen (z.B. Leitlinienentwicklung, Evidence-Based Medicine und Infection Control) zu antizipieren,

- realistische Zielerwartungen für die Evaluation zu formulieren, und

- auf dieser Basis die politischen Entscheider realistisch zu informieren.

Neben der Abstimmung mit den dominierenden Vergütungsanreizen und der Formulierung realistischer Zielerwartungen ist es die zentrale Aufgabe des Rahmenkonzeptes, Kriterien für die Priorisierung des Handlungsbedarfs zu definieren Jedes Gesundheitssystem, jedes Vergütungssystem hat seine charakteristischen Qualitätsprobleme. Deswegen ist es auch nicht sinnvoll, Qualität und Patientensicherheit ,an sich“ "zu untersuchen, zu verbessern, zu bearbeiten, sondern man muss dies im Rückgriff auf die zugrundeliegenden Strukturen tun. Das US-amerikanische Gesundheitssystem, um ein Beispiel zu nennen, hat als Crundproblem die ausufernden Kosten und den mangelnden Zugang zur Gesundheitsleistung: fee for service auf der einen Seite, Managed Care auf der anderen. Das deutsche Gesundheitssystem ist wie viele andere westliche Gesundheitssysteme durch seine Mengen- und Prozedurenorientierung, die Vernachlässigung von chronischen Erkrankungen und Prävention, die ausgeprägte Sektorierung sowie den Anbieterbezug charakterisiert ist (s. „fünf Ziele“, Kap. 1.4)

Am Beispiel $\mathrm{P} 4 \mathrm{P}$ kann man den Zusammenhang zwischen der qualitätsverbessernden Intervention und den dominierenden Vergütungsinstrumenten gut beschreiben (s. Miller 2009; Rosenthal 2008). Die Mengenorientierung stellt sie einerseits das Hauptargument für die Einführung von $\mathrm{P}_{4} \mathrm{P}$ dar („wir vergüten Menge statt Qualität, daher muss P4P kommen“), ist aber gleichzeitig eines der Haupthindernisse für dessen Wirksamkeit, weil in einem mengenorientierten System die Opportunitätskosten besonders hoch sind - als Alternative zur Investition in Qualitätsverbesserung und Dokumentation gilt immer: „ein Fall geht noch“ (ausführliche Darstellung einschl. Risikoaversion und Diskontierung s. Kap. 8.6.1). Sektorale Gliederung sowie 
die Dominanz der Akuterkrankungen und operativen Medizin über Prävention wirken sich in gleicher Weise aus.

\subsection{Qualität als Systemeigenschaft}

Qualität und Patientensicherheit in der Gesundheitsversorgung werden in Deutschland traditionell als individuelle Leistung, bestenfalls als Leistung eng umgrenzter Teams angesehen. Diese Einschätzung geht einher mit der Überschätzung der medizinischen Gesundheitsleistung, die - wenngleich von entscheidender Bedeutung - jedoch nur unter Berücksichtigung des gesamten Kontextes Erfolg haben kann (s. Kap. 1.3). Diese Kontextleistung zu untersuchen, ist Aufgabe der Versorgungsforschung.

Es war die Thematik Patientensicherheit, die erstmalig der Ansicht zum Durchbruch verhalf, dass die Kontextleistung von entscheidender Bedeutung sein könnte, weil klar wurde, dass noch so viel Anstrengung des Einzelnen es nicht wahrscheinlicher machen würde, dass Leistungen und Prozesse im Gesundheitswesen fehlerärmer und daher für den Patienten schadensfreier ablaufen würden (s. Report „To Err Is Human“ des IOM (Kohn et al. 1999).)

Diese Überschätzung der individuellen Verantwortung, die (um es nochmals zu betonen) andererseits auch nicht unterschätzt werden darf (denn individuelle Verantwortung wird es immer geben und ist auch nicht zu relativieren), die aber nicht hinreichend ist, um eine gute, patientenorientierte Gesundheitsversorgung zu realisieren, diese Überschätzung zeigt sich auch auf zahlreichen anderen Ebenen (s. Abb. 46):

- So ist es eine typische Reaktion von Personen und Organisationen im Gesundheitswesen, bei Bedrohung des Geschäftsmodells, also bei Infragestellung der Wertschätzung der erbrachten Leistung, von dieser Leistung noch mehr anzubieten, sich noch mehr anzustrengen, statt darüber nachzudenken, wie man sie besser organisieren kann oder ob man das Leistungsspektrum verändern sollte. Der eingängige Begriff, der sich

Erfolg und Instabilität der Umwelt: die Anstrengung und das Können des Einzelnen sind hinreichend (Hamsterrad).



$$
\begin{aligned}
& \rightarrow \text { Patientensicherheit } \\
& \rightarrow \text { Arbeitsgestaltung } \\
& \rightarrow \text { Organisationserfolg } \\
& \rightarrow \text { Gesundheitspolitik }
\end{aligned}
$$

Abb. 46 Die Versorgungsleistung setzt sich aus der Gesundheitsleistung und der Kontextleistung zusammen (vgl. Pfaff und Schrappe 2011, S. 21) 
für diese Situation eingebürgert hat, ist das sog. Hamsterrad, in dem sich die Betreffenden gefangen fühlen, noch schneller, noch mehr. Der Arzt dehnt, im typischen Fall, seine Arbeitszeit ins Unerträgliche aus, statt zu überlegen, wie es (z.B. durch Verteilung von Verantwortung) auch anders zu organisieren wäre. In der Konsequenz bilden sich Arbeitsvorstellungen aus, die z.B. mit den Erwartungen der jüngeren Generation an ihre Arbeitsbedingungen nichts mehr zu tun haben.

- Auf einer weiteren Ebene ist dieses Hamsterradphänomen auf sektoraler Ebene zu beobachten. Die Vergütungssysteme, allen voran die fallpauschalierenden Systeme wie das DRG-System, fördern zu allererst die sektorale Optimierung, vor allem auf der Ebene der Mengenentwicklung. Die sektoralen Budgets sind gedeckelt, daher ist eine Leistungsausweitung kaum möglich, gleichzeitig müssen die einzelnen Player, gerade wenn sie nur eine grenzwertige Auslastung ihrer Kapazitäten vorweisen können, eine immerwährende Mengenausweitung betreiben, um mindestens ihren Platz halten zu können. Die Qualität der Versorgung, als das was als Versorgung beim Patienten ankommt, spielt kaum eine Rolle mehr, es geht nur um das Überleben der Institution. Um ein Beispiel aus dem Krankenhausbereich zu nennen: Im Jahr 2005, also nach Abschluss der DRG-Einführung, wurden in 2.139 Krankenhäusern bei einer Auslastung von 74,6\% 16,5 Mill. Patienten behandelt, im Jahr 2012 in 2.017 Krankenhäusern bei einer Auslastung von 77,4\% bereits 18,6 Mill. Patienten; die Verweildauer fiel in dieser Zeit von 8,7 Tagen auf 7,6 Tage ab. Diese Entwicklung ist dramatisch, nicht nur dass mehr als 2 Mill. Patienten mehr behandelt wurden, was innerhalb von nur 7 Jahren eine Zunahme um weit mehr als 10\% bedeutet, und was natürlich die Frage aufwirft, ob es wirklich denkbar ist, dass sich die Morbidität der Bevölkerung im gleichen Zeitraum so verändert hat, dass eine derartige Zahl von zusätzlichen Patienten stationär behandelt werden musste. Denn gleichzeitig steht unverändert jedes vierte Krankenhausbett leer - wäre es wirklich nur eine Morbiditätsproblematik, wäre die Verweildauer konstant geblieben und die Auslastung der Betten auf zwischen 85 und 90\% angestiegen. Es ist also nicht die Morbidität, sondern das Hamsterrad, was wir hier beobachten können, und was auch in jedem Gespräch mit Krankenhausärzten, in jeder Versammlung mit Krankenhausärzten zu hören ist. Man verkürzt die Verweildauer aus finanziellen Gründen und um aufnahmebereit zu sein für die unausweichlich notwendige Fallzahlausweitung.

- Aber damit nicht genug, es gibt noch eine dritte Ebene, auf der das Kleinteilige, das Hochtourige dominiert, nämlich die Gesundheitspolitik. Die Übertragung auf diese Ebene mag auf den ersten Blick etwas holprig erscheinen, aber auch hier regiert die „individuelle Anstrengung “ in Form von kleinteiligen Regelungen zur Abwehr von Finanzierungslücken, zur Abwehr von offensichtlichen Regelungslücken im Hinblick auf Qualität (z.B. Hygieneskandale der letzten Jahre), zur Abwehr von 
vermuteten Qualitätsdefiziten als Folge von Veränderungen im Vergütungssystem (Fallpauschalen beim Gesundheitsstrukturgesetz 1993, DRGs in der GKV 200o-Reform etc.). Als Konsequenz haben wir eine einzigartige Vielfalt von Einzelregelungen in den unterschiedlichsten Teilen der Sozialgesetzbücher, die allesamt vom guten Willen getragen sind, das kann man gar nicht abstreiten - aber eine übergeordnete Entwicklungslogik, man könnte auch sagen: eine tragfähige Strategie, eine Melodie fehlt (s. auch Kap. 1.1). Das heißt eben nicht, um Missverständnissen vorzubeugen, dass auf gesundheitspolitischer Ebene alles geregelt werden muss (und das wäre bei der regionalen Organisation der Gesundheitsversorgung auch nicht möglich), aber ein direction pointing im Sinne des governance-Konzeptes wäre schon notwendig (vgl. Kap. 9.5).

Es gibt internationale Vorbilder, zumindest die conceptual frameworks in den USA (Crossing the Quality Chasm des IOM [2001]) und in Großbritannien (The NHS Outcome Framework 2013/2014 [NHS 2013B]) sind hier zu nennen: „Trying harder will not work. Changing Systems of care will" (IOM 2001, S. 4f; s. Kap. 8.3.1). Wie in Kapitel 4.5 schon dargelegt, erstaunlich ist hier weniger der systemtheoretische Kontext, in dem diese Konzepte eingebettet sind, sondern die Konsequenz und Langfristigkeit, mit der diese über Jahrzehnte entwickelt und letztendlich auch umgesetzt und publiziert wurden (s. Kap. 4.5, s. auch IOM 2007, CMS 2011B, CMS 2012, Chien und Rosenthal 2013).

Akzeptiert man die These, dass Qualität (und Patientensicherheit) eine Systemeigenschaft sind, ist die Konsequenz, dass diese Thematik auch auf Systemebene weiterentwickelt werden muss - im Sinne strategischer Vorgaben, im Sinne des direction pointing, in der frühzeitigen Identifikation unerwünschter Effekte und im Sinne von Rahmenbedingungen (s. Kap. 9.5). Als Beispiel für solche strategischen Vorgaben könnte die Integration der Versorgung auf regionaler Ebene gelten, ohne die die Versorgung einer alternden und regional abnehmenden Bevölkerung nicht möglich ist (direction pointing), wobei Monopolsituationen vermieden werden müssen. Es sind Area-Indikatoren notwendig (s. Kap. 2.4.9), die die Qualität der Versorgung auf regionaler Ebene beschreiben, um resultierende negative Entwicklungen zu verhindern. Vorgaben und Rahmenbedingungen dieser Art werden handlungsleitend und ermutigend auf die anderen Ebenen des Systems einwirken, die regionalen Körperschaften, Organisationen, die einzelnen an der Versorgung beteiligten Personen, denn sie kennen die langfristige Entwicklung, können ihr Tun danach ausrichten und sich damit auseinandersetzen. Wie wichtig solche Vorgaben sein können, ist in Untersuchungen durchaus bereits dargelegt worden (Rosenthal 2007, s. Kap. 9.5). 


\subsection{Qualität und Sicherheit: Operationalisierung}

Kein direction pointing kann jedoch zum Ziel führen, keine Systembetrachtung hat einen Sinn, wenn man Qualität (und Sicherheit) nicht operationalisieren kann. In Deutschland begibt man sich hierbei in Auseinandersetzungen zur Frage der Nutzung administrativer Daten, zur Problematik Ergebnis- vs. Prozessindikatoren, zum Verständnis des Indikatorenkonzeptes. Diese Fragen sind zweifellos wichtig, bleiben aber an der Oberfläche, denn das Hauptproblem in der Operationalisierung des Qualitätsbegriffes liegt in der Frage, welche Qualitätsaspekte man in die Betrachtung einbezieht, und welche Zielvorstellungen man dabei verfolgt. Wie in Kapitel 1.3 und 1.4 bereits ausgeführt und als grundlegende Systematik für diese Arbeit genutzt, handelt es sich dabei um drei Dimensionen, die den sog. dreidimensionalen Orientierungsrahmen bilden (s.u.):

- Dimension der sechs Qualitätsperspektiven (s. Kap. 1.3): von welchem Erkenntnisinteresse geht man aus, welche Qualitätsaspekte werden einbezogen, bleibt z.B. man bei der Betrachtung von Diagnosen und Prozeduren stehen oder bezieht man Systemfragen (Koordination), Nutzenbetrachtungen oder Patientenerfahrungen mit ein;

- Morbiditätsdimension(s. Kap. 1.4): welche Erkrankungen werden einbezogen, inwieweit wird das zukünftige Krankheitsspektrum, das durch chronische Mehrfacherkrankungen charakterisiert ist, und die Prävention berücksichtigt;

- Integrationsdimension (s. Kap. 1.4): inwieweit werden die strukturellen Entwicklungsnotwendigkeiten des Gesundheitssystems mit einbezogen, insbesondere bezüglich der Integration der Versorgung und in der Überwindung der Sektorierung, oder mit anderen Worten: inwieweit wird Qualität nicht nur als ein defensives Konzept zur Neutralisierung von Fehlentwicklungen verstanden, sondern als ein aktives Konzept zur Bewältigung von zukünftigen Aufgabenstellungen.

Bereits auf der Ebene der diagnose- und prozedurenbezogenen Qualitätssicherung (s. Kap. 2.1) werden erhebliche Lücken im Spektrum der Erkrankungen deutlich, die von der bisherigen Qualitätssicherung abgedeckt werden. Selbst wenn man eigentlich darüber diskutieren müsste, warum Morbidität, die Weiterentwicklung des Gesundheitssystems und die Qualitätsperspektiven (z.B. Patientenerfahrungen) außer acht gelassen werden, muss man zunächst doch feststellen, dass bereits auf dieser einfachsten Ebene der Diagnosen und Prozeduren deutliche Defizite bestehen. Diese Defizite sind nur historisch zu begründen - die Sonderentgelte und Fallpauschalen des Gesundheitsstrukturgesetzes 1993 gaben z.B. einen solchen engen Rahmen vor. Das Festhalten an dieser Sichtweise kann man nur als Betriebsblindheit des (hochtourig laufenden) Systems mit seiner Mesoebene Gemeinsamer Bundesausschuss verstanden werden, feststeht aber, dass sie hochgradig dysfunktional sind. 
Wir diskutieren fortwährend über die Gesundheitsversorgung der Zukunft, in der die chronischen und Mehrfacherkrankungen einer immer älter werdenden Bevölkerung im Mittelpunkt stehen, und unsere Qualitätssicherung beschränkt sich so gut wie ausschließlich auf operativ zu behandelnde Akuterkrankungen und Transplantationsmedizin, auch im Bereich der Kardiologie werden praktisch nur kardiologisch interventionelle Eingriffe thematisiert (s. Kap. 2.1). Dieses Spektrum hat mit den konservativ zu behandelnden, z.T. sogar nur zu begleitenden(!) Erkrankungen, die wir im Gesundheitswesen der Zukunft vor allem zu vergegenwärtigen haben, nichts zu tun, da kann es an Deutlichkeit gar nicht fehlen. Jede Weiterentwicklung der Qualitätssicherung muss sich ausnahmslos daran messen lassen, dass hier die Entwicklung gestoppt wird, man eine erkrankungsbezogene Problemanalyse durchführt und man erst dann fortfährt, wenn man die Krankheitsbilder identifiziert hat, mit denen wir es in der Weiterentwicklung unseres Gesundheitswesens in Zukunft zu tun haben. Es ist kein Problem, die Cholezystektomie und Herztransplantation weiter mitlaufen zu lassen, aber der Schwerpunkt der Entwicklung muss auf den chronischen Erkrankungen aus den konservativen Fachgebieten einschließlich der Koordination der Sektoren und Leistungsanbieter (s.u.) liegen.

Bei der Betrachtung der Morbiditätsdimension darf man jedoch nicht stehen bleiben, sondern die Dimension der aktiven Nutzung der Qualität im Rahmen der Weiterentwicklung des Gesundheitssystems einbeziehen, insbesondere der Integrationsdimension (s. Abb. 47). Die führenden Problematiken des deutschen Gesundheitssystems (Sektorierung, Akuterkrankung, kein Anreiz für Prävention etc.) sind in diesem Gutachten bereits mehrfach angesprochen worden (s. Kap. 1.4). Der Ausgangspunkt dieser Überlegungen ist ein sektoriertes, im Hamsterrad der sektoralen Optimierung gefangenes System, der Zielpunkt ist ein auf die Bedürfnisse der regionalen Versorgung zuge-

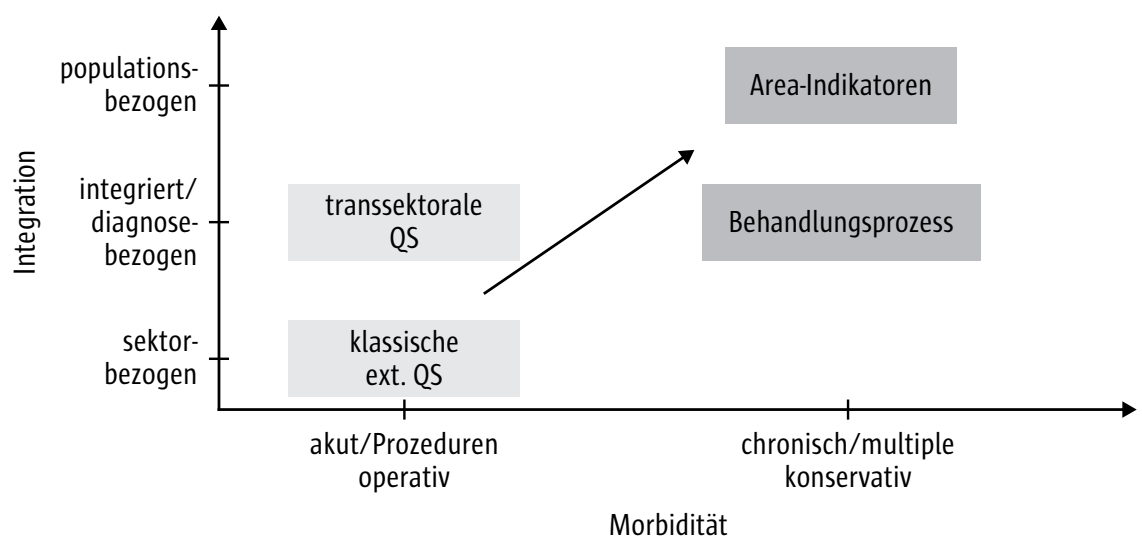

Abb. 47 Zweidimensionale Betrachtung von Qualität: Die Morbiditätsdimension wird kombiniert mit der strukturellen Integrationsdimension. 
schnittenes System, das über Anreize zur Prävention verfügt und trotzdem der Gefahr der Vorenthaltung von Leistungen (Zugangsverschlechterung durch den Prototyp Managed Care; s. Kap. 2.4.9) beherrscht. Auf dem Weg zu dieser Zielvorstellung wird die diagnose- und prozedurenbezogene Qualitätssicherung verlassen, die Sektor-übergreifende Qualitätssicherung (möglichst bald) wegen der Konservierung der Sektorproblematik überwunden, eine integrierte Perspektive eingenommen und zunehmend im Rahmen einer weitergehenden Diskussion um regionale Versorgungskonzepte Qualitätskonzepte entwickelt, die z.B. mittels area-Indikatoren (s. Kap. 2.4.9) den Einsatz solcher Konzepte unterstützen. Um es an dieser Stelle nochmals zu sagen: dies ist der Abschied von einer rein defensiven Nutzung des Qualitätskonzeptes („das Schlimmste verhüten“) und die Hinwendung zu einem Qualitätskonzept, das sich als aktives Entwicklungsinstrument versteht.

Der dritte Punkt betrifft nun die hier so bezeichneten Qualitätsperspektiven, die die Sichtweise auf Qualität verdeutlichen und hier der Übersichtlichkeit wegen noch einmal genannt seien (s. Kap. 1.3):

- gesellschaftliche Perspektive (Versorgung auf Populationsebene),

- Ebene der Effizienz (Nutzen der Behandlungsmethode und Allokation),

- Patientenperspektive (Selbstbestimmung, Sicherheit und Koordination),

- professionelle Perspektive (Autonomie, Garantenstellung),

- institutionelle Perspektive (organisatorische Umsetzung),

- wissenschaftliche Perspektive (Deskription und Hypothesenbildung).

Der neutrale, systematische Qualitätsbegriff, der sich auf die „Anforderungen“ bezieht, wird nicht verlassen, aber wegen seiner Tendenz zur Beliebigkeit in der konkreten Umsetzung ergänzt. Es geht eben nicht um beliebige Anforderungen, sondern um ganz definierte Sichtweisen, unter denen dann Anforderungen formuliert werden können und sollen. Traditionellerweise wird die diagnose- und prozedurenbezogene Qualitätssicherung z.B. von Nutzenbetrachtungen abgegrenzt, man erkennt diese Trennung auch an der Etablierung des neuen Institutes für Qualitätssicherung und Transparenz im Gesundheitswesen vom Institut für Qualität und Wirtschaftlichkeit im Gesundheitswesen $(\mathbb{1} 137 \mathrm{a}$ [neu] nach dem Finanzstruktur- und Qualitätsweiterentwicklungsgesetz (GKV-FQWG) vom 6.5.2014; s. Kap. 7.2) - beide Institute firmieren unter dem Begriff der „Qualität“, aber man leistet sich die Doppelstruktur, weil nicht erkannt wird, dass beide Qualitätsperspektiven gleichermaßen Bestandteil eines sinnvollen Qualitätskonzeptes sind.

Letztlich kommt man daher in Erweiterung des zweidimensionalen Konzeptes (s. Abb. 47) zu einem dreidimensionalen Orientierungsrahmen, der die drei Dimensionen Morbidität (Chronizität/Prävention), Struktur (Integration und Koordination) und Qualitätsperspektiven vereint (s. Abb. 48, vgl. Kap. 1.4). Der „Ball“ muss sozusagen von der vorderen, unteren und linken Ecke in die hintere, obere und rechte Ecke; die horizontale Morbiditätsdimension umfasst 


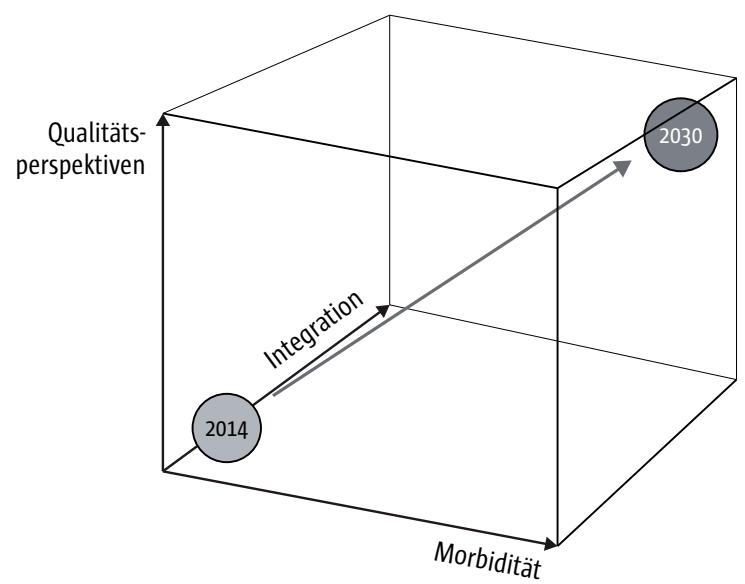

Abb. 48 Dreidimensionaler Orientierungsrahmen (s. Text, s. Kap. 1.4)

sowohl die Betreuung von chronisch Kranken als auch die Prävention des Auftretens von chronischen Erkrankungen sowie deren Episoden, die vertikale Achse das Maß, in dem die unterschiedlichen Qualitätsperspektiven einbezogen werden, und die in die Tiefe reichende Perspektive die Integration des Leistungsgeschehens (Aufhebung der Sektorierung).

An diesem Punkt, und wirklich erst jetzt, kommen die evergreen-Fragen zum Zug, mit denen sich die Diskussion in Deutschland in unsinniger Vordergründigkeit immer wieder beschäftigt:

- wieviel Qualität, wieviel Patientensicherheit? (s. Kap. 1.3)

- Prozess- vs. Ergebnisqualität (s. Kap. 2.4.5)

- administrative („Sozial“-)Daten vs. klinische surveillance-Daten (s. Kap. 2.4.6)

- quantitative Erfassung vs. Indikatoren mit Vorhersagefunktion (s. Kap. 2.4.3).

Zur Frage Prozess- vs. Ergebnisindikatoren ergibt sich demgegenüber jedoch ein ganz neuer Aspekt, der unter Kapitel 2.4.5 noch nicht genannt wurde: es sind nicht nur die Fragen des Präventionsaspektes, der Risikoadjustierung und kleiner Gruppengrößen, die die Ergebnisindikatoren gegenüber den Prozessindikatoren ins Hintertreffen geraten lassen. Vielmehr treten Patientenerfahrungen umso mehr in den Mittelpunkt der Analyse, umso mehr der Integrationsaspekt und Fragen der Informationsübermittlung, Beteiligung von Patienten und der Koordination der Behandlung als wichtig erkannt werden. Prozessindikatoren dieser Art müssen in Teilen zwar noch entwickelt werden, sie werden aber die Herausforderung der Zukunft sein - natürlich neben gut validierten Ergebnisindikatoren, die z.B. bei regionalen Versorgungsstrukturen zur Geltung kommen müssen, um die Outcome-Qualität beurteilen zu kön- 
nen; die Gruppengröße spielt hier dann vielleicht auch gar nicht mehr eine so große Rolle wie bei der vergleichenden Betrachtung von einzelnen Leistungsanbietern.

Auch die Bedeutung von administrativen Daten in der Qualitätssicherung wird sich ändern. Wenn man das Szenario von regionalen Netzen mit Versicherungsfunktion diskutieren möchte, die nur noch wenige Abrechnungsdaten nach außen übermitteln müssen und diese nunmehr nur zur Regelung der internen Leistungsbeziehungen verwenden, wird die Bereitschaft, diese Daten zu Zwecken der Qualitätssicherung zur Verfügung zu stellen oder sie überhaupt deswegen erst zu generieren, stark abnehmen. Will man nicht die Gefahr eingehen, dass solche Managed Care-ähnlichen Versorgungsstrukturen als black boxes im Gesundheitswesen gelten, muss man von außen die Verpflichtung zur Qualitätssicherung aufrechterhalten, allerdings unter der begleitenden Bedingung, dass es nicht mehr mit Verweis darauf, „die Daten seien sowieso da“, geschehen kann.

Natürlich wird es auch in Integrierten Versorgungssystemen die Notwendigkeit geben, unerwünschte Ereignisse durch quantitative Verfahren zu erfassen. Allerdings wird die Rolle der „wirklichen“ Indikatoren mit Vorhersagefunktion noch wichtiger werden als heute, denn in der dann noch höheren Komplexität der Versorgungssysteme muss man besonders auf den Lerneffekt (s. Kap. 8.4.4) der Systeme setzen, und dies ist nur durch Indikatoren möglich, die in präventiver Sicht Problembereiche aufzeigen und zum Gegenstand einer Gegensteuerung machen.

\subsection{Kontext: Institutionen, Verhaltensänderung, System}

Bei der Entwicklung eines Rahmenkonzeptes, dessen Anforderungen in Kapitel 9.1 formuliert wurden, kann zwar auf die genannten Beispiele aus anderen Gesundheitssystemen Bezug genommen werden (Crossing the Quality Chasm des IOM (2001) bzw. The NHS Outcome Framework 2013/2014 [NHS 2013B]), trotzdem müssen die Eigenschaften des deutschen Gesundheitssystems im Vordergrund stehen. Die Faktoren, die in das Rahmenkonzept einfließen müssen, sind außerordentlich zahlreich, heterogen und in ihren Wechselwirkungen vielfältig:

Rahmenkonzept: Zugang

Organisationstheorie: Expertenorganisation (professional bureaucracy) (s. Kap. 8.2)

- Systemtheorie: Komplexe Systeme (s. Kap. 8.3)

- Verhaltensänderung (s. Kap. 8.4)

- lerntheoretische Modelle (s. Kap. 8.4.2)

- soziale Wahrnehmung (s. Kap. 8.4.3) 


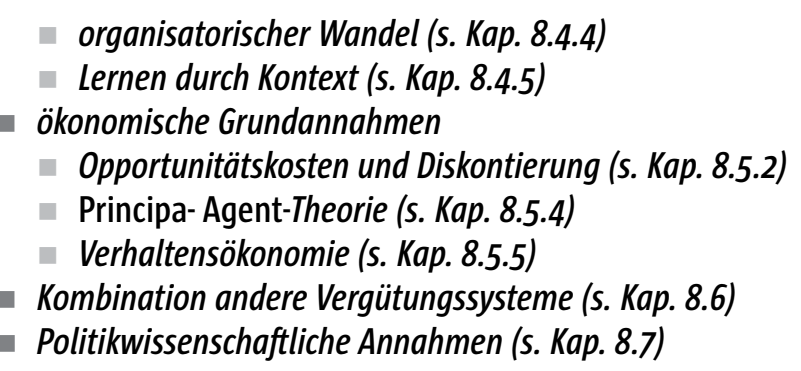

Das in dieser Arbeit entwickelte Konzept stützt sich zunächst auf das aus der Organisationstheorie stammende Konzept der Expertenorganisation (Spezialistenbürokratie, professional bureaucracy) und auf die aus der Systemtheorie stammende Komplexitätstheorie. Diese Konzepte weisen in bestimmten Bereichen Parallelen auf und werden hier unter dem Arbeitsbegriff der komplexen professionellen Systembürokratie zusammengefasst (Definition s. Kap. 8.3.3):

- beide Konzepte betonen die Unsichtbarkeit der (gleichwohl vorhandenen) internen Regeln, in der Expertenorganisation in Form der dezentralen Vernetzung der Fachexperten, im komplexen System grundsätzlich zwischen allen Teilen des Systems;

- die Teile des Systems und die Experten im operativen Kern der Expertenorganisation verfügen über ein hohes Maß an Autonomie, gleichzeitig sind sie lern- und anpassungsfähig und neigen zur Selbstorganisation (in der Expertenorganisation auf fachlichen Ebene in Abgrenzung zur Managementebene), unvorhergesehene und in ihrer Ausprägung stark differierende Reaktionen der Organisation bzw. des Systems sind die Regel;

- beide Konzepte können hochinnovativ sein, jedoch sind die Innovationen in Art, Menge und Zeitpunkt nicht vorhersehbar und können von außen nicht mit voraussagbarem Erfolg angestoßen werden, insbesondere wenn es sich um Prozessinnovationen handelt („Innovationspara doxon“). Jegliche Versuche, hier Einfluss zu nehmen, wird als Reduktion der Autonomie und als innovationsfeindlich interpretiert;

- beide Konzepte akzeptieren Unsicherheit: der Experte in der Expertenorganisation wird immer improvisieren müssen („Lehrer vor der Klasse“), die Komplexitätstheorie sieht Unsicherheit, Spannung und Paradoxie (im Gegensatz zu linearen Modellen) sogar als konstituierendes Bestandteil von Systemen an und erklärt eine Reduktion dieser Erscheinungen als aussichtslos oder nur in Ansätzen erreichbar. Diese „intrinsische Unsicherheit “, die einerseits als Toleranz gegenüber Unsicherheit sicherlich eine adäquate Eigenschaft darstellt, denn sie schützt vor irreführenden linearen Konzepten, ist andererseits gerade beim Thema Qualität und Patientensicherheit ein kritischer Punkt. 
Hinzu kommt, dass nicht nur der durch die professionelle komplexe Systembürokratie beschriebene Kontext, sondern auch die Intervention $\mathrm{P} 4 \mathrm{P}$ selbst komplexer Natur ist (sog. „,doppelte Komplexität“, zum Begriff vgl. Shojania 2013).

Zusammenfassend kann man von drei Basismerkmalen sprechen, die bei der Implementierung von $\mathrm{P}_{4} \mathrm{P}$ in einem entwickelten Gesundheitssystem eine Rolle spielen: Innovationsparadoxon und intrinsische Unsicherheit des Systems sowie doppelte Komplexität von Instrument und Kontext.

Vor diesem Hintergrund ist die Rolle der unterschiedlichen Konzepte der Verhaltensänderung in einer komplexen professionellen Systembürokratie zu klären (s. Kap. 8.4). Lerntheoretische Ansätze greifen zu kurz, da Einstellungen, professionellen Loyalitäten und sozialen Rollen, ganz abgesehen von ökonomischen und politischen Faktoren (s.u.), eine sehr große, konkurrierende Rolle zukommt. Konzepte der sozialen Wahrnehmung thematisieren zusätzlich die Einstellungsebene und das Rollenverständnis, wichtig vor allem bezüglich des Themas Professionalismus, denn die traditionellen Rollenbilder geraten unter Druck und müssen sich an veränderte Bedingungen und veränderte Aufgaben innerhalb des Gesundheitssystems anpassen. Verhaltensänderung durch organisatorischen Wandel, die auf einen unabhängigen Wissens- und „Wertevorrat“ der Organisation setzen, der einer Veränderung durch Lernprozesse zugänglich ist, scheitern an dem mangelnden Austausch zwischen Organisation/System und Experten. Die kontextbezogenen Theorien sind am weitesten gespannt und stellen daher die Favoriten dar, insbesondere da es mit dem VBP-Programm in den USA und dem QOL-Programm in Großbritannien potente Beispiele gibt, in denen man bei der Implementierung von P4P auf diese Konzepte setzt. Allerdings bedürfen diese Methoden des sozialen Marketings einer langfristigen, strategisch aufgebauten Planung, die von der politischen Seite aus gesteuert werden muss und in ihrer Dauer weit über eine Legislaturperiode hinausreichen.

Zusammenfassend muss festgehalten werden, dass in der Vergangenheit bei den Diskussionen um P4P ebenso wie um Leitlinienimplementierung oder Evidence-Based Medicine sehr stark auf lerntheoretische und Modelle der sozialen Wahrnehmung (einschließlich Professionalismus) gesetzt wurde. Für eine erfolgreiche Umsetzung müssen jedoch Konzepte des organisatorischen Wandels und kontextbezogene Modelle im Vordergrund stehen.

Allerdings ist die Einführung von komplexen Instrumenten zur Qualitätsverbesserung wie $\mathrm{P} 4 \mathrm{P}$ nicht allein auf der Ebene organisatorischer Konzepte oder de individuellen Verhaltensänderung möglich, insbesondere wenn man sich die hohen Erwartungen vergegenwärtigt, die im Gesundheitswesen an diese Instrumente gestellt werden. Als weitere Voraussetzungen werden in der vorliegenden Arbeit somit noch ökonomische und Vergütungsfragen (s. Kap. 8.5 und 8.6) sowie politikwissenschaftliche Konzepte (s. Kap. 8.7) diskutiert: 
- Die Höhe der qualitätsbezogenen Vergütung und das System der monetären Kopplung an Qualitätsindikatoren und müssen kritisch gewertet werden, insbesondere unter Berücksichtigung der Opportunitätsund Grenzkosten.

- Zusätzlich ist die Diskontierung der P4P-Vergütung zu berücksichtigen, falls die Zahlungen nicht zeitnah, sondern mit Zeitverzug erfolgen, u.U. sogar später als die Vergütung eventueller zusätzlicher Fallerlöse.

- Aus den Argumentationszusammenhängen der behavioural economics ist zusätzlich zu den Opportunitätskosten und der Diskontierung eine mögliche Risikoaversion einzubeziehen, wenn für den Leistungserbringer nicht sicher ist, ob er eine qualitätsbezogene Vergütung erhält (und er sich dann für die „sichere“ zusätzliche Fallerbringung entscheidet). Dies kann z.B. dann der Fall sein, wenn bei einer relativ sortierten Rankingliste die relative Position genauso von der Performance der Mitbewerber wie von der eigenen Position abhängt. Genauso sind ein adäquates framing und die Berücksichtigung der Tendenz zur Überschätzung zeitnaher, kleiner Zahlungen von Wichtigkeit.

- Von großer Bedeutung ist die Tatsache, dass $\mathrm{P} 4 \mathrm{P}$ nicht als alleiniges Vergütungssystem eingeführt werden kann, sondern immer in ein allgemeines Vergütungssystem eingebettet ist. Dieses zugrundeliegende Vergütungssystem weist immer Anreizfunktionen auf, die unter Umständen der qualitätsbezogenen Vergütung durch P4P zuwiderlaufen können (z.B. DRG-System und starker Mengenanreiz). Man muss die Elemente einer $\mathrm{P} 4 \mathrm{P}$-Vergütung folglich sehr vorsichtig und gezielt in die allgemeine „Vergütungslandschaft“ integrieren.

Zusammenfassend sind aus ökonomischer Sicht vor allem Opportunitätskosten, Grenzkosten, Diskontierung bzw. Zeitachse und Aspekte der Risikoaversion zu bewerten, außerdem ein optimales framing. Von entscheidender Bedeutung ist jedoch die Abstimmung mit dem dominierenden Vergütungssystem, bei solchen mit einem starken Mengenanreiz ist es nicht sinnvoll, P4P und andere Instrumente des Qualitätswettbewerbes bei Erkrankungen einzusetzen, die für eine Mengenausweitung anfällig sind. Stattdessen sollten Prozessindikatoren aus dem Bereich der Koordination und Behandlung von chronischen Erkrankungen im Vordergrund stehen.

\subsection{Rolle von Politik und Gesetzgebung}

Die genannte Kritik an modernen neokorporatistischen, „neoinstitutionalistischen“ Politikkonzepten wie Governance ist nur allzu berechtigt, denn so wichtig es ist, die Akteure mit in Themensetzung und Entscheidungsbildung einzubeziehen, um eine bessere Umsetzbarkeit zu gewährleisten, so wichtig ist es auch, dass die Politik i.S. der legislativen Entscheidungen, der exekutiven Umsetzung und der juriskativen Kontrolle sich nicht selbst entmachtet 
(vgl. Mayntz 2010). Eine solche vorauseilende Selbstentmachtung verbietet sich nicht nur wegen der Gewährleistung der Gesundheitsversorgung (s.o.), sondern weil ohne politischen Druck bewegt sich nichts (Moran u. Scanlon 2013). Auch wenn Veränderungen auf der Ebene der Individuen und Institutionen vor Ort ablaufen, das „System“ ist deswegen nicht aus der Verantwortung. Die politische Sphäre hat ihre spezifischen Aufgaben zu erfüllen, muss hierzu ihre spezifischen Methoden anwenden und hat dazu ihre spezifischen Strukturen zu nutzen.

Der Rückgriff auf die politische Theorie findet nun im Zusammenhang mit qualitätsverbessernden Instrumenten wie Public Reporting und $\mathrm{P}_{4} \mathrm{P}$ seine $\mathrm{Be}$ gründung darin, dass für die Einführung und die erfolgreiche Umsetzung eines Instrumentes wie $\mathrm{P} 4 \mathrm{P}$ auch auf politischer Ebene Weichenstellungen notwendig sind, die die vorangehenden Überlegungen zur Rolle der Organisation, der individuellen Verhaltensänderung, der Komplexität des Systems und der ökonomischen Grundannahmen ergänzen. Immerhin, das darf nicht vergessen werden, handelt es sich gerade bei $\mathrm{P} 4 \mathrm{P}$ um einen massiven Eingriff in das Gesundheitswesen: der Fokus wird von der Menge (volume) auf die Qualität der Gesundheitsversorgung gelegt, ein - falls erfolgreich - geradezu paradigmatischer Wandel. Im Einzelnen müssen auf der politischen Ebene folgende vier Bedingungen thematisiert und implementiert werden:

- Direction pointing

- strategische Ziele setzen

- negative Auswirkungen kontrollieren

- Rahmenbedingungen schaffen

- Direction pointing: Dieser Begriff stammt aus dem IOM-Werk „Crossing the Quality Chasm“ von 2001 und bringt es auf den Punkt: die Richtung vorgeben, neben „prohibitions“ und „resource of permission providing“ eines der drei simple rules, die für eine Intervention in komplexen Systemen geeignet sind (Plsek 2003). Die system- oder komplexitätstheoretische Provenienz dieses Begriffes ist offensichtlich: da die internen Regeln nicht bekannt sind, die Auswirkungen von Interventionen nicht vorhersehbar sind und das System darüber hinaus zur spontanen Selbstorganisation neigt, wäre alles sinnlos, was über eine Richtungsanzeige hinausgeht. Diese Richtungsanzeige kann sich auch auf die in komplexen Systemen vorhandenen Attraktoren beziehen, die als End- oder Zwischenzustände dienen und - obwohl von außen nicht direkt sichtbar - eine relative Stabilität bieten (s. Kap. 8.3.3).

Man muss dem Konzept nicht einmal folgen, allerdings gibt es empirische Daten, die belegen, dass die Akteure im Gesundheitswesen eine solche „Richtung“ durchaus wünschen und unterstützen. In der Befragung der early adopters von $\mathrm{P}_{4} \mathrm{P}$ in den USA gaben diese an, dass sie $\mathrm{P}_{4} \mathrm{P}$ trotz des Fehlens überzeugender Wirksamkeitsnachweise weiter verfolgen wollen, weil es besser sei, für Qualität bezahlt zu werden als für die Menge, und weil sie sich davon langfristig Transparenz für die Patien- 
ten versprechen (Rosenthal 2007). Die Richtungsvorgabe hat in diesem Sinne eine wichtige kohärenzverstärkende Wirkung, ähnlich wie die Organisationskultur in Organisationen: das Große Ganze muss sichtbar sein. Im Rahmen des Value-Based Purchasing (VBP)-Programms in den USA wird eine solche Vorgabe bespielhaft gemacht: „to foster joint clinical and financial accountability“, die Verantwortung auf klinischer und finanzieller Ebene stärken (CMS 2011B).

Natürlich muss das direction pointing in einer glaubwürdigen Form geschehen. Dies ist leichter gesagt als getan, denn die Angehörigen der Gesundheitsberufe haben ein feines Gespür, sie fassen solche Vorgaben durch die Politik schnell als „Manager-Sprech“ auf. Logik und Sprache auf der Ebene von Politik (und Management) sind anders als am Krankenbett, gerade wenn es - wie bei $\mathrm{P} 4 \mathrm{P}$ - um wettbewerbsbezogene Elemente geht (s. Kap. 8.2.2, auch Edwards 2005). Insofern ist die immer wiederkehrende Bezugnahme auf mangelnde Qualität dann schwierig, wenn andere, gleichzeitig wirksame Anreize (z.B. Mengenanreiz) dominant sind und die Qualitätserwartung konterkarieren. Es ist daher z.B. zu diskutieren, ob - allen Ökonomisierungs-Debatten zum Trotz - in diesem Kontext das Konzept des value, also das Verhältnis von Qualität und Kosten, als Zielgröße für $\mathrm{P}_{4} \mathrm{P}$ am Ende nicht glaubwürdiger ist als die Zielgröße Qualität allein. In den USA wird es im Value-Based-PurchasingProgramm (VBP) so praktiziert. Diese Annahme erscheint auf den ersten Blick paradox, aber von außen auf Geld angesprochen zu werden wird von den Angehörigen der Gesundheitsberufe unter Umständen weniger befremdlich erlebt als auf die Qualität ihrer Arbeit.

In jedem Fall ist die Diskussion zu führen: wie kann ein authentisches direction pointing durch die politische Ebene aussehen? Eine „stumme Gesundheitspolitik“ ist auf jeden Fall nicht die Lösung. Als Beispiel sei hier auf eine bislang erstaunlicherweise wenig beachtete Möglichkeit zur Förderung und Verstärkung des Qualitätsgedankens hingewiesen, nämlich die verstärkte Förderung der Ausbildung in den Bereichen Ethik, Ökonomie und Qualität/Patientensicherheit für die Angehörigen der Gesundheitsberufe. Man kann sich auf den Standpunkt stellen, dass der Fortschritt, den das deutsche Gesundheitswesen in punkto Qualität und Patientensicherheit zu einem definierten Zeitpunkt in der Zukunft erreicht hat, gut durch die Integration dieser Aspekte in die Ausbildung beschrieben werden kann (Schrappe 2013). Nebenbei: damit hätte die Integration dieser Themen in die Ausbildung die Funktion eines klassischen Indikators.

- Strategische Ziele setzen: Wie aus den vorangehenden Kapiteln bereits deutlich geworden ist, können qualitätsverbessernde Instrumente wie Public Reporting und P4P keine Wunder vollbringen, insbesondere können dominante Anreize wie z.B. den Mengenanreiz durch das DRG-System nicht ausgeglichen werden. Diese Instrumente bieten aber bei gezieltem Einsatz die Gelegenheit, zentrale Problemfelder eines Gesund- 
heitssystems anzugehen - jedes Gesundheitssystem hat seine charakteristischen Stärken und Schwächen. Im deutschen Gesundheitssystem wären hier neben der Orientierung an Mengen und (operativ zu behandelnden) Akuterkrankungen vor allem die Koordinationsdefizite durch die ausgeprägte Sektorierung zu nennen (s.o., z.B. Schoen et al. 2011). Die Voraussetzung ist jedoch, dass man sich vor (!) Implementierung nach strategischen Gesichtspunkten ein Bild davon macht, welche dieser grundlegenden Probleme in welcher Reihenfolge anzugehen sind (Berenson et al. 2013). Diese strategischen Überlegungen können dann Indikatoren, meist Prozessindikatoren ohne Risikoselektion und ohne Aufhebung der Informationsasymmetrie (s.o.), identifizieren, die dann gerade für die Versorgung chronisch und mehrfach Erkrankter sowie die Versorgung älterer Patienten eine große Bedeutung haben. Andere Indikatoren können sich z.B. mit der Unterversorgungsproblematik in ländlichen Regionen beschäftigen, man muss hier jedoch diskutieren, ob die angestrebten Ziele nicht eher durch direkte Unterstützung und Einzelleistungsvergütung anzugehen wären.

Diese strategischen Ziele zu setzen, ist eine genuin politische, gestaltende Aufgabe, die zwar die Unterstützung der Selbstverwaltungspartner bedarf, in ihrer Ausgestaltung jedoch in den Händen des Cesetzgebers liegen muss, nicht zuletzt deswegen, weil evtl. nicht unerhebliche Steuermittel aufgebracht werden müssen. Auch in den USA werden solche strategische Ziele derzeit intensiv diskutiert, z.B. die bessere Koordination über die Sektorgrenzen (Daughtridge et al. 2014) oder die optimale Versorgung der Bevölkerung, insbesondere der underserved populations (Rosenthal et al. 2007; Brown et al. 2014).

- Negative Auswirkungen kontrollieren: Gerade wenn ein neues gesundheitspolitisches Instrument eingesetzt wird, besteht die Verantwortung des Gesetzgebers in der Vermeidung bzw. Kontrolle etwaiger unerwünschter Auswirkungen. Diese Kontrolle kann in Zusammenarbeit mit Verbünden und anderen Partnern des Gesundheitswesens zusammen erfolgen, die Letztverantwortung liegt jedoch auf der Ebene der Politik. Die möglichen unerwünschten Auswirkungen der Einführung von P4P wurden zusammenfassend in Kapitel 6.5 aufgeführt und diskutiert. Ganz im Vordergrund stehen Probleme des Zugangs (sozial benachteiligte Bevölkerungsgruppen, Kontinuität der Versorgung, Risikoselektion, vgl. z.B. Ryan 2013) und der Verzerrung durch Datenmanipulation.

- Rahmenbedingungen schaffen: Die Rahmenbedingungen der Einführung von Public Reporting oder $\mathrm{P}_{4} \mathrm{P}$ sind Gegenstand dieses Gutachtens (Rahmenkonzept). Es handelt sich dabei um Systemfaktoren und Umsetzungsparameter, die die möglichen Auswirkungen dieser Instrumente direkt oder indirekt fördern oder konterkarieren, und sie liegen in der Verantwortung der gesetzgeberischen Ebene, sie sind, um diesen Ausdruck im alten Sinne zu gebrauchen, ein politisches Problem (Miller 2009). Es handelt sich dabei nicht nur um reine Motivations- oder öko- 
nomische Faktoren, wie z.B. konkurrierende ökonomische Anreize, die den Anreizen der P4P-Vergütung entgegengesetzt sind (z.B. Einzelleistungsvergütung). Dies ist ein ganz entscheidendes Problem, ohne Frage, aber negative Rahmenbedingungen können auch darin bestehen, dass bei Umsetzung und Spezifikation eines Instrumentes wie P4P Fehler gemacht werden. Da es hier im wahrsten Sinne des Wortes um Geld geht, kann man z.B. nicht erwarten, dass auf der Ebene der „Systembürokratie“, also der Selbstverwaltung, eine zwanglose Einigung zu den anzuwendenden Indikatoren zustande kommt, eher ist eine langdauernde Blockade mit langfristiger Ergebnislosigkeit zu erwarten. Die Governance findet also dort ihre Grenzen, wo Interessen zurückgeschnitten werden müssen, Beispiele für ein Versagen der „Verhandlungsdemokratie“ gibt es zu Genüge (z.B. Mindestmengenindikatoren im Krankenhausbereich). Zu den Rahmenbedingungen gehört es auch, die Komplexität des Geschehens nicht aus den Augen zu verlieren. Es reicht nicht, an einer Schraube zu drehen. Die Implementierung eines Instrumentes wie $\mathrm{P}_{4} \mathrm{P}$ muss immer im Kontext anderer Interventionen gesehen werden, nicht nur der Rahmen, der durch die zugrundeliegenden Vergütungssysteme (z.B. DRG) gegeben wird, sondern auch die wirtschaftliche Situation der Krankenhäuser, die Veränderungen in der Trägerschaft, die Sektorierung des Gesundheitswesens, die Morbidität etc.

Natürlich ist die Ausgestaltung der Institutionen im Bereich der Selbstverwaltung (z.B. Gemeinsamer Bundesausschuss) rational, weil nur so eine bessere Partizipation erreicht werden kann, aber es besteht die Gefahr der Blockade und Suboptimierung (Mayntz 2010). Hinzu kommt die Tendenz jeder Institution, Funktion mit Selbsterhaltung und Selbstbeschäftigung zu verwechseln und letztendlich notwendige Entwicklungen zu blockieren. Es muss daher verstärkt darüber nachgedacht werden, wie die „Zivilgesellschaft“, damit ist hier gemeint: die nicht mit dem Gesundheitswesen verbundenen Gesellschaftsgruppen, Berufe, Verbände, Unternehmen, in das Thema Qualität und Sicherheit eingebunden wird, ein Ansatz, der in den USA z.B. mit der Leapfrog-Initiative erfolgreich beschritten wird (Galvin et al. 2005; Moran u. Scanlon 2013). 


\section{Zusammenfassung:}

Aus politikwissenschaftlicher Sicht sind bei der heute vorherrschenden Tendenz zu Governance-Konzepten (s. Kap. 8.7) vier Aspekte von Bedeutung:

- Es muss ein Rahmen erkennbar sein, der für die angestrebten Veränderungen einen Interpretations- und Sinnzusammenhang darstellt (direction pointing).

- Ein sinnvoller Einsatz von P4P ist ohne strategische Überlegungen nicht möglich, damit die optimalen und entscheidenden Ansatzpunkte für eine Weiterentwicklung des Gesundheitssystems identifiziert und angegangen werden können.

- Von politischer Seite muss erkennbar Verantwortung und Vorsorge getragen werden für etwaige negative Effekte der qualitätsorientierten Vergütung.

- Es müssen günstige Rahmenbedingungen geschaffen werden, z.B. durch Vorgaben zur Gestaltung der institutionellen Kooperationen, die für die Umsetzung verantwortlich sind. Die stärkere Einbeziehung der zivilgesellschaftlichen Ebene, die mit dem Gesundheitssystem primär nicht verbunden, jedoch an Qualität und Sicherheit genuin interessiert ist, ist zu diskutieren. 